(1]

Department of Experimental, Diagnostic and Specialty Medicine, University of Bologna, Bologna, Italy.

\title{
GPs Meet Rare Lung Disorders Task Force factsheet: pulmonary arterial hypertension
}

\section{Definition}

Pulmonary hypertension $(\mathrm{PH})$ is a haemodynamic and pathophysiological condition defined as an increase in mean pulmonary arterial pressure $\geq 25 \mathrm{mmHg}$ at rest as assessed by right heart catheterisation. PH can be found in multiple clinical conditions as shown in the box below.

Pulmonary arterial hypertension (PAH; group 1) is a clinical condition characterised by the presence of precapillary $\mathrm{PH}$ in the absence of other causes of precapillary $\mathrm{PH}$, such as $\mathrm{PH}$ due to lung diseases, chronic thrombo-embolic $\mathrm{PH}$ or other rare diseases. $\mathrm{PAH}$ includes different forms that share a similar clinical picture and virtually identical pathological changes of the lung microcirculation as shown in the box below.

\section{Prevalence}

The prevalence of $\mathrm{PAH}$ in the general population in Europe is approximately 1 in 20,000.

\section{Clinical manifestations}

The symptoms of $\mathrm{PAH}$ are non-specific and include breathlessness, fatigue, weakness, angina, syncope and abdominal distension. Symptoms at rest are reported only in very advanced cases. The physical signs of PAH include left parasternal lift, an accentuated pulmonary component of second heart sound, a pansystolic murmur of tricuspid regurgitation, a diastolic murmur of pulmonary insufficiency and a right ventricular third heart sound. Jugular vein distension, hepatomegaly, peripheral
Cite as: Galiè N, Manes A, Palazzini M, et al. GPs Meet Rare Lung Disorders Task Force factsheet: pulmonary arterial hypertension. Breathe 2015; 11: 233-236

\section{Updated clinical classification of pulmonary hypertension}
1. Pulmonary arterial hypertension $(\mathrm{PAH})$
$1^{\prime}$ : Pulmonary veno-occlusive disease and/or pulmonary capillary haemangiomatosis
2. Pulmonary hypertension due to left heart disease
3. Pulmonary hypertension due to lung diseases and/or hypoxaemia
4. Chronic thromboembolic pulmonary hypertension
5. PH with unclear and/or multifactorial mechanisms 


\section{Updated clinical classification of pulmonaryarterial hypertension}

\section{Pulmonary arterial hypertension (PAH)}

1.1 Idiopathic PAH

1.2 Heritable

1.2.1 BMPR2 mutation

1.2.2 ALK1, endoglin (with or without hereditary haemorrhagic telangiectasia)

1.2.3 Unknown

1.3 Drugs and toxins induced

1.4 Associated with

1.4.1 Connective tissue diseases

1.4.2 HIV infection

1.4.3 Portal hypertension

1.4.4 Congenital heart disease

1.4.5 Schistosomiasis

1.4.6 Chronic haemolytic anaemia

1.5 Persistent pulmonary hypertension of the newborn

1 ' Pulmonary veno-occlusive disease and/or pulmonary capillary haemangiomatosis.

oedema, ascites and cool extremities characterise patients in a more advanced state. Lung sounds are usually normal. The examination may also provide clues as to the cause of $\mathrm{PH}$. Telangiectasia, digital ulceration and sclerodactly are seen in scleroderma, while inspiratory crackles may point towards interstitial lung disease. The stigmata of liver disease such as spider naevi, testicular atrophy and palmar erythema should be looked for. If digital clubbing is encountered, an alternative diagnosis such as congenital heart disease or pulmonary venoocclusive disease should be sought.

\section{Diagnosis}

The evaluation process of a patient with suspected $\mathrm{PH}$ requires a series of investigations intended to confirm the diagnosis, clarify the clinical group of $\mathrm{PH}$, the specific aetiology within the PAH group, and evaluate the functional and haemodynamic impairment. The various investigative tests can be combined in a diagnostic algorithm (figure 1) which, for practical purposes, can be divided into four phases:

\section{Suspicion}

Clinical suspicion of $\mathrm{PH}$ should be aroused when there are symptoms such as breathlessness without overt signs of specific heart or lung disease, in cases of screening in predisposing conditions or in cases of incidental findings.

\section{Detection}

The detection of $\mathrm{PH}$ requires investigations able to confirm the diagnosis, e.g. clinical examination, ECG, chest radiograph and transthoracic echocardiography.

\section{Group identification}

The next step is the identification of the clinical group. This is accomplished by the use of essential investigations such as pulmonary function tests, arterial blood gases and ventilation-perfusion lung scans. In particular circumstances, additional tests can be performed such as high-resolution computed tomography (CT) of the chest, contrast-enhanced spiral CT of the lung and pulmonary angiography.

\section{Evaluation}

After the diagnosis of PAH (clinical group 1), additional investigations are required for the exact identification of the type and for the assessment of exercise capacity and haemodynamics.

\section{Referral}

The purpose of a referral centre is to undertake assessment and investigation of all causes of $\mathrm{PH}$, routinely manage appropriate patients with $\mathrm{PAH}$ including the provision of specific drug therapy, work with other healthcare providers to obtain best outcomes for patients, and undertake audit, research and education. Since, in general, centres with a high volume of patients tend to obtain best outcomes, referral centres will need to have sufficient patients on chronic therapy and new referrals to warrant this status.

\section{Treatment and follow-up}

The therapy of $\mathrm{PAH}$ patients cannot be considered as a mere prescription of drugs, but is characterised by a complex strategy which includes the evaluation of severity, supportive and general measures, the assessment of vasoreactivity, the estimation of 


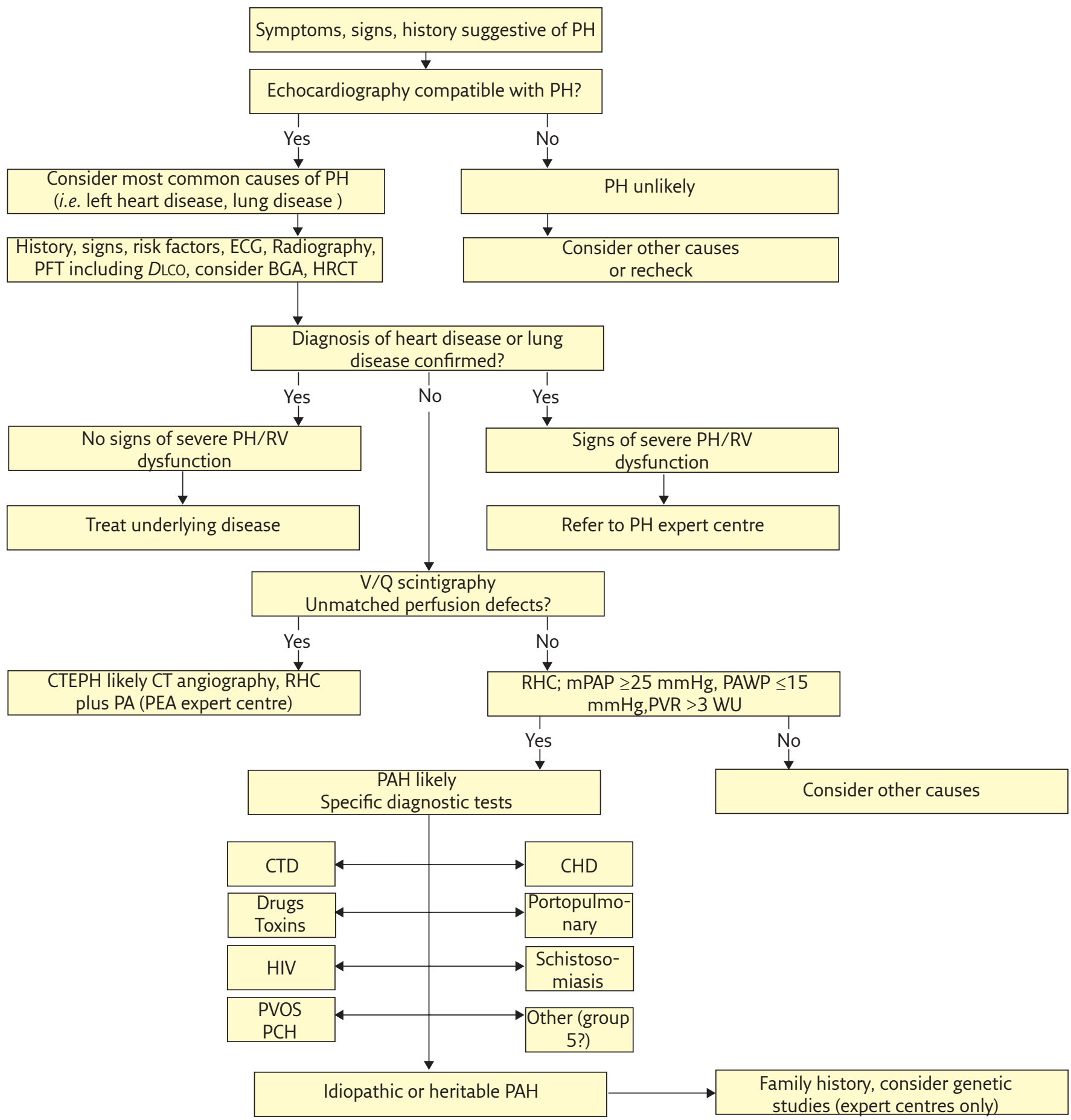

Figure 1 ECG: electrocardiogram; PFT: pulmonary function testing; DLCO: diffusion capacity of the lung for carbon monoxide; BGA: blood gas analysis; HRCT: high-resolution computed tomography; RV: right ventricle; VIQ: ventilation/perfusion; CTEPH: chronic thromboembolic pulmonary hypertension; RHC: right heart catheter; PA: pulmonary angiography; PEA: pulmonary endarterectomy; mPAP: mean pulmonary artery pressure; PAWP: pulmonary arterial wedge pressure; CTD: connective tissue disease; CHD: congenital heart disease; PVOD: pulmonary veno-occlusive disease; PCH: pulmonary capillary haemangiomatosis. Reproduced from [3] with permission from the publisher.

efficacy and combination of different drugs plus it is strongly recommended that patients with PAH interventions.

Three classes of drugs have been specifically approved for PAH (prostanoids, endothelin receptor antagonists and phosphodiesterase (PDE)-5 inhibitors. Guanylate cyclase stimulators affect the nitric oxide pathways like PDE-5 inhibitors. Due to the complexity of the treatment options available, are referred to a specialist centre.

A treatment algorithm for $\mathrm{PAH}$ patients has been proposed. The treatment algorithm is not appropriate for patients with $\mathrm{PH}$ from other causes and in particular for patients with $\mathrm{PH}$ associated with left heart diseases or with parenchymal lung diseases. General measures and initiation of supportive 


\section{Key Points}

- Focus on the difference between PH (a frequent pathophysiological condition present in many diseases) and PAH (a rare disease for which specifically approved drugs are available).

- Suspect PAH in case of breathlessness in particular in young individuals and in presence of predisposing conditions.

- Adopt a rigorous diagnostic algorithm (as shown in figure 1).

- After diagnosis of PAH, refer the patients to an experienced centre for the adoption of the treatment algorithm.

therapy need to be initiated after PAH diagnosis. Acute vasoreactivity testing should be performed and high-dose calcium channel blockers therapy performed as appropriate. Non-responders to acute vasoreactivity testing who are in World Health Organization (WHO)/ New York Heart Association (NYHA) functional class II should be treated with an endothelin receptor antagonist, a PDE-5 inhibitor or a guanylate cyclase stimulator. Non-responders to acute vasoreactivity testing, or responders who remain in (or progress to) WHO/NYHA functional class III should be considered candidates for treatment with an endothelin receptor antagonist, a PDE-5 inhibitor, a prostanoid or a guanylate cyclase stimulator.

As head-to-head comparisons among different compounds are not available, no evidence-based first-line treatments can be proposed. In this case, the choice of the drug is dependent on a variety of factors, including the approval status, the route of administration, the side-effect profile, patient preferences, costs and physician's experience. Some authors still use first-line i.v. epoprostenol in WHO/NYHA functional class III patients, due to its demonstrated survival benefits. Continuous i.v. epoprostenol may be considered as first-line therapy for WHO/NYHA functional class IV PAH patients because of the demonstrated survival benefit in this subset. Subcutaneous and i.v. treprostinil has also been approved for the treatment of WHO/ NYHA functional class IV patients. Both endothelin receptor antagonists and PDE-5 inhibitors are considered as a second line for severely ill patients, in WHO/NYHA functional class IV patients initial combination therapy may also be considered. In case of inadequate clinical response to first-line therapy, sequential combination therapy can be considered. Combination therapy can either include a prostanoid plus an endothelin receptor antagonist or an endothelin receptor antagonist plus a PDE-5 inhibitor or a prostanoid plus a PDE-5 inhibitor. Guanylate cyclase stimulators may be utilised instead of a PDE- 5 inhibitor. The combination of these two compounds is contraindicated. Balloon atrial septostomy and/or lung transplantation are indicated for PAH with inadequate clinical response despite optimal medical therapy or where medical treatments are unavailable.

This factsheet adapted from [1].

\section{Conflict of interest}

None declared

\section{Further reading}

1. Galiè N, Hoeper MM, Humbert M, et al. Guidelines for the diagnosis and treatment of pulmonary hypertension. Eur Respir J 2009; 34: 1219-1263.

2. Galiè N, Hoeper MM, Humbert M, et al. Guidelines on diagnosis and treatment of pulmonary hypertension: The Task Force on Diagnosis and Treatment of Pulmonary Hypertension of the European Society of Cardiology and of the European Respiratory Society. Eur
Heart J 2009; 30: 2493-2537.

3. Hoeper MM, Bogaard HJ, Condliffe R, et al. Definitions and Diagnosis of Pulmonary Hypertension. J Am Coll Cardiol 2013; 62: 25 Suppl., D42-D50.

4.www.escardio.org/guidelines-surveys/esc-guidelines/ Pages/pulmonary-arterial-hypertension.aspx

5. www.phaeurope.org 